\title{
IKO: a five actuated DoF upper limb exoskeleton oriented to workplace assistance
}

\author{
Felix Martinez*, Aron Pujana-Arrese, Iban Retolaza, Irantzu Sacristan, Jon Basurko and Joseba Landaluze \\ Ikerlan Technological Research Centre, Arizmendiarrieta-2, E20500 Arrasate/Mondragon, Basque Country (Spain)
}

(Received 7 October 2008; final version received 6 March 2009)

\begin{abstract}
IKerlan's Orthosis (IKO) is an upper limb exoskeleton oriented to increasing human force during routine activity at the workplace. Therefore, it can be considered as a force-amplification device conceived to work in collaboration with the human arm and implementing biomimetic principles. The aim of the proposed design is to find the best compromise between maximum reachable workspace and minimum moving mass, which are the key factors for obtaining an ergonomic, wearable exoskeleton. It consists of five actuated degree of freedom (DoF) to move the human arm and three non-actuated DoF between the back and shoulder to allow relative displacement of the sterno-clavicular joint. Conventional electrical motors are used for most of the DoF and pneumatic muscles for one of them (forearm rotation). Power transmission is based on Bowden cables. This paper presents the IKO design, the mechanical structure of a first prototype and the redesign process from an aesthetic point of view. Controller set-up and control strategies are also shown, together with dynamic performance from experimental results.
\end{abstract}

Keywords: upper limb exoskeleton; upper limb orthosis; workplace assistance; wearable robot

\section{Introduction}

The human body can be considered as the best robot ever designed, but it is limited by muscle strength and fatigue. Musculoskeletal disorders (MSDs) constitute the most common work-related health problem in Europe and affect millions of workers. Across the European Union (EU)-27 nations, $25 \%$ of workers complain of backache and 23\% report muscular pains (Podniece 2007). MSDs are caused mainly by manual work, heavy physical work, awkward and static postures, repetition of movements and vibration. They can provoke intense pain to the point where it becomes difficult or impossible to carry out daily tasks. The economic cost estimated by the members of the European Agency for Health and Safety at Work (EU-OSHA, FACTS) related to muscular-skeletal difficulties ranges between $0.5 \%$ and $2 \%$ of the gross domestic product (GDP) (Podniece 2007).

Tackling MSDs means taking action at the workplace. Preventative measures can be taken initially and rehabilitation techniques for workers who already have MSDs can also be applied, but in the end, technical assistance also requires consideration. Intelligent Assist Devices (IADs) are mechatronic devices whose aim is to help the user carry out daily tasks that require a certain effort. These efforts may not be too great, but if they are carried out frequently (either in a repetitive or a habitual manner in the workplace or in a domestic environment), they can eventually cause problems, tiredness and even injury. Therefore, IADs can be defined as machines designed not to replace the worker but to help carry out the work more safely and efficiently. Under that definition, cobots (collaborative robots) could also be considered, but they are actually a form of IAD. In more specific terms, a cobot is a robot for direct interaction with the operator in a shared workspace (Akella et al. 1999) and is normally used to transport or handle materials. At Ikerlan, IAD is considered as a wearable device (exoskeleton): users wear the device on their body. Newer concepts, such as the ergonomic versions (weight, comfort and aesthetics), manoeuvrability (monitoring of human movements), sturdiness and safety for the user when working etc. need to be taken into account. Therefore, an exoskeleton, 'body amplifier' or orthosis is a biomechatronic system worn by a person in such a way that the physical interface enables the direct transfer of mechanical power and exchange of information (Ruiz et al. 2006). In this case, exoskeletons are considered for amplifying human force, with the user as a control-order generator and most of the force supplied by the exoskeleton. The user feels only a scaled-down part of the external force required for feedforward purposes and control set-point adjustment.

In this context, Ikerlan has designed an upper limb IAD named IKerlan's Orthosis (IKO), a wearable exoskeleton aimed at helping the user perform a routine activity at the workplace.

From the review of the current state of the art in upper limb power amplification exoskeletons, they can be divided

${ }^{*}$ Corresponding author. Email: felix.martinez@ikerlan.es 
by taking into account two criteria: the transmission of the forces at the end of the mechanical chain to the ground or the body (external or internal force) and the kinematical chain concept. Force-amplification exoskeletons normally require an external force system, while functional compensation applications can implement internal force concepts (Pons 2008). End-effector-based exoskeletons are independent of joint alignments, but are not safe or ergonomic (Schiele and van der Helm 2006) and can damage human joints by overloading. Another design paradigm for ergonomic exoskeleton design is that the kinematical structure must not copy the structure of the adjacent human limb (Schiele and van der Helm 2006).

Pons (2008) presents and emphasises the features of some of the most relevant upper limb exoskeletons identified in the literature. Applications range from teleoperation to rehabilitation and assistance for the disabled or the elderly. As a conclusion, considering the exoskeleton as an $\mathrm{IAD}$, there are few designs available. Moreover, most recent developments have focused on reducing total exoskeleton weight and, more specifically, on reducing the weight of the mobile parts. This concerns not only ergonomic issues, such as being able to move within the natural workspace without limiting the operator's range of motion, but also its dynamic performance and wearability (Ruiz et al. 2006). At the same time, as they are actuated devices, their design must take into account safety criteria, portability and ease-of-use capabilities.

This paper presents IKerlan's Orthosis (IKO), an exoskeleton with five actuated DoF for human upper limb power amplification purposes. For ergonomics and kinematical compatibility purposes, four additional nonactuated (passive) DoF are implemented: three to avoid macro-misalignments of the shoulder centre position, and one to avoid micro-misalignment of the elbow rotation centre with reference to the wrist. In addition to conventional electric motor actuators, the design is based on two key elements: pneumatic muscles and Bowden cable-drive power transmission. According to Klug et al. (2006) this enables a considerable reduction of moving masses and required power, resulting in high-performance design. The proposed design can also be used for internal or external force systems. Finally, the paper gives preliminary results on the corresponding dynamic response of the conventional and non-conventional actuators that are used.

\section{Selection of DoF}

\subsection{Specifications}

As mentioned above, the main objective was to design a wearable powered exoskeleton to help the user work more safely and efficiently. Taking into account this fact and the state-of-the-art review, the following initial requirements were established:
1. An upper limb IAD to help the user perform a routine activity at the workplace.

2. A wearable, lightweight exoskeleton; however, the power devices might not be wearable.

3. It must be able to replicate the human arm's workspace in front of the person to the highest degree possible with the hand axis in vertical position.

4. It is assumed that the user can use his hand to grasp objects.

5. The user may grasp and hold a maximum weight of 10 $\mathrm{kg}$.

6. If possible, non-conventional actuators should be used and biomimetic concepts applied.

\subsection{Kinematical chain configuration}

Biomechanical upper arm models in the literature (NASA 2008) are simplified to seven DoF (see Figure 1): three at the shoulder $(1,2,3)$; two at the elbow $(4,5)$; and two at the wrist $(6,7)$. With regard to ergonomic aspects, the kinematical chain must allow movement within the natural workspace without limiting the operator's range of motion.

For the industrial application being considered (e.g. handling objects in a vertical position on a workbench), the natural human workspace is in front of the person, between the waist and the head. In order to minimise the DoF of the exoskeleton, all the combinations with four and five DoF were analysed and the following conclusions were drawn (Martinez et al. 2007):

1. The three DoF of the shoulder (1-3) cannot be dissociated without avoiding natural movements.

2. Elbow flexion-extension movement (4) is crucial to maximise workspace.

3. Configurations with four DoF provide a very narrow workspace.

4. Two optimal solutions are obtained with five DoF: 1-3 and 4-5; or 1-3 and 4-7. The option with two DoF at the elbow joint (4-5) offers a workspace below shoulder

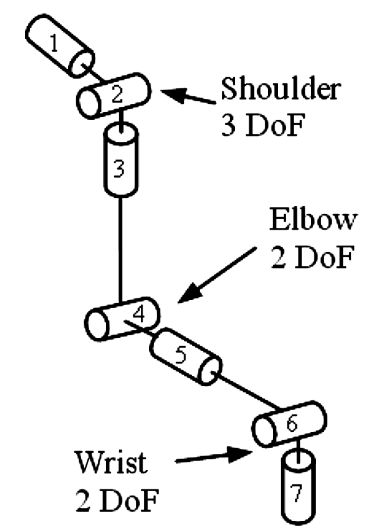

Figure 1. Human arm kinematics. 

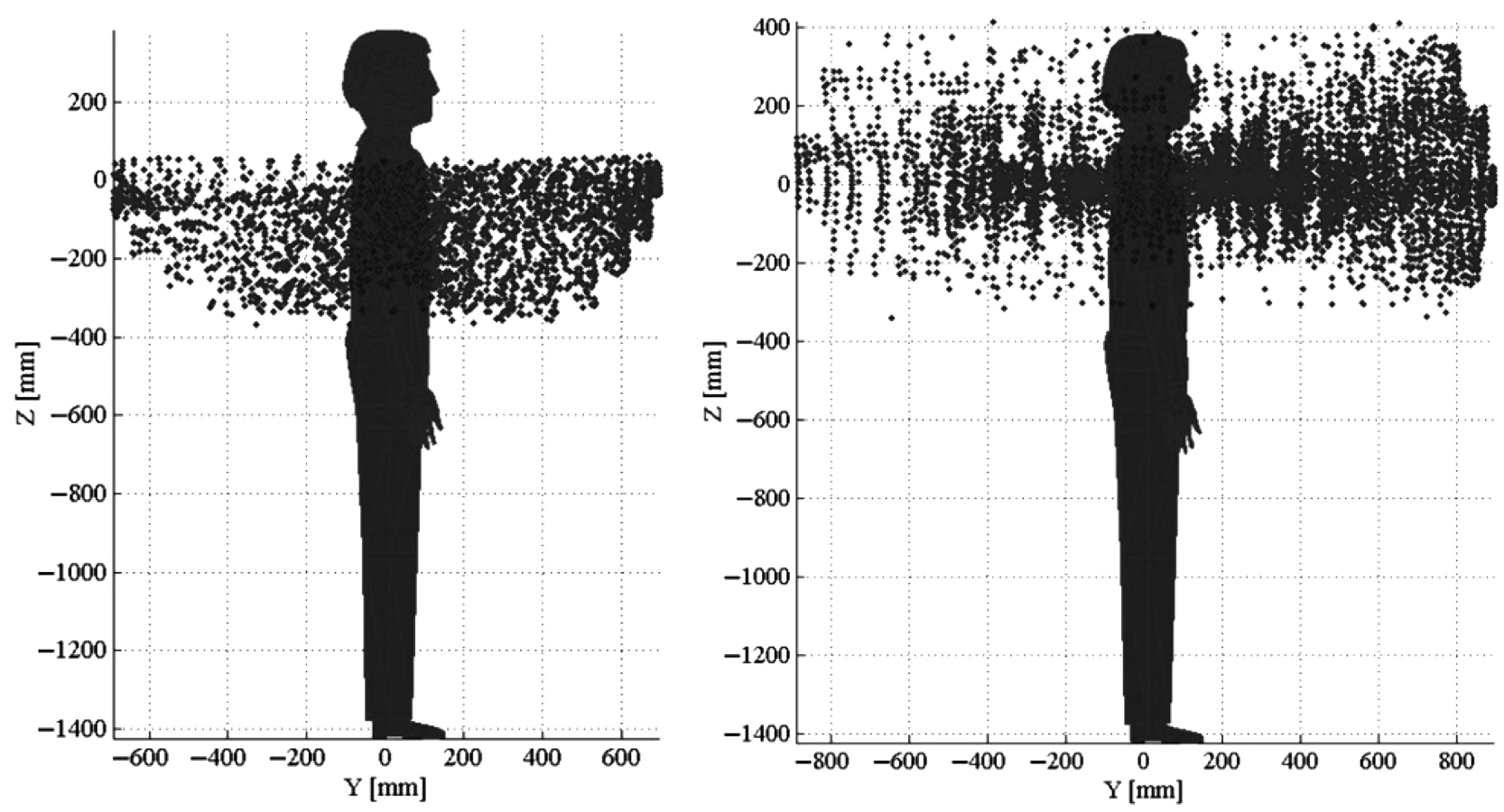

Figure 2. Theoretical workspace: (a) two DoF at elbow (4-5); (b) one DoF at elbow and the other at wrist (4-7).

height and therefore closer to the workbench compared to option (4-7), as shown in Figure 2.

From this study, the option with five DoF (three at the shoulder and two at the elbow, cf. Figure 3) was chosen for the design described in this paper. Wrist DoF is not considered for this application, assuming that the user can use his hand to grasp the objects.

The configuration of the DoF at the shoulder is a key point from the viewpoint of ergonomics, range of movement and force transmission between exoskeleton and the user's body. The human shoulder behaves like a spherical joint, with four rotational DoF, but has been simplified to three DoF, placed in series, as proposed in the literature by NASA (2008), with a common centre of rotation. This provides a reachable work space similar to that of the human arm, reduces interference with other body parts and allows a simple transmission of forces between the exoskeleton and the arm.

Nevertheless, with this simplification, singular points appear at certain placements that can be overcome by changing the initial orientation of the first and second DoF in order to change the areas where singularities appear. For IKO, a Cartesian configuration of the axis has been chosen, taking into account that the system is designed to work in a workbench environment. Planed tasks mostly required movements of the second to fifth axis, with no movements of the shoulder around a vertical absolute axis.

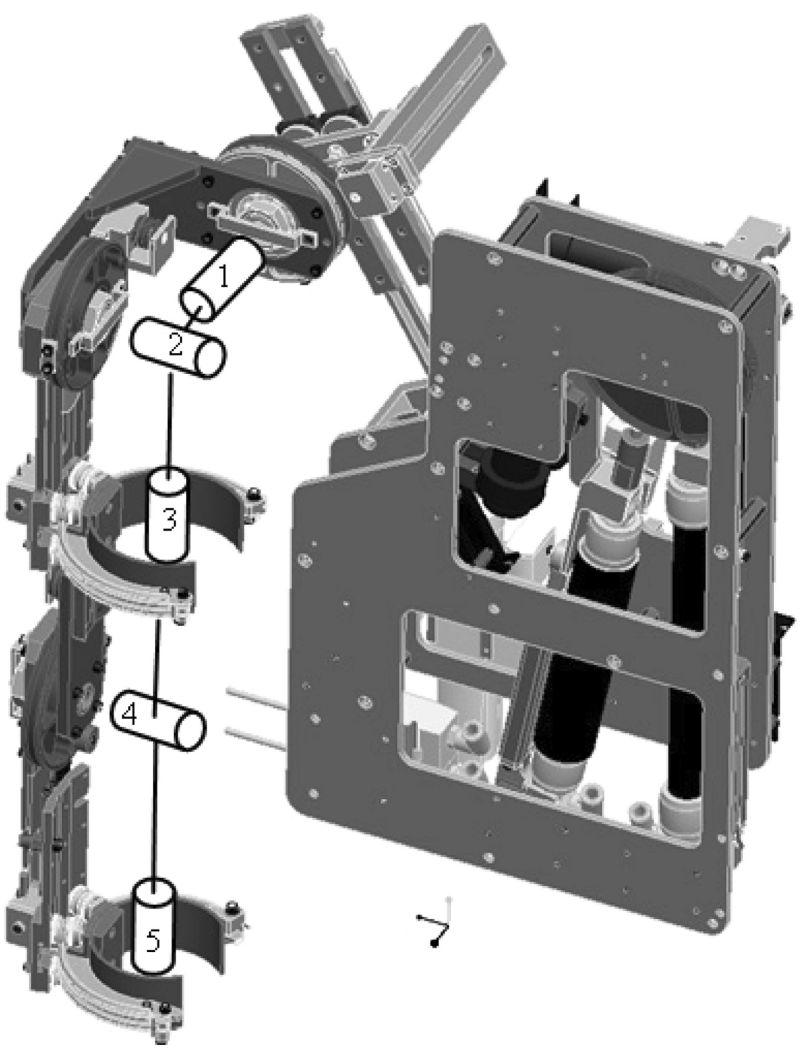

Figure 3. Kinematical joint configuration and selected DoF. 
Therefore, the first of the rotations corresponds to the abduction movement, the second to the flexion movement and the third to the rotation movement. This latter rotation is obtained by means of a circular guide, as proposed by Perry and Rosen (2006). The distance between the second and third DoF can be adjusted to different wearers (Figure 3 ). The DoF of the elbow is designed in a similar way: the fourth DoF corresponds to elbow flexion and the fifth to forearm rotation. The distance between these two DoF can be altered to avoid micro-misalignment between the elbow and the wrist rotation centre.

The proposed configuration is attached to the human body at three points: on the back (the reference point for the movements), the arm (near the elbow), and the forearm (above the wrist).

As the proposed kinematical chain does not take into account the movement of the shoulder centre and as a result of sterno-clavicular movement, additional DoF must be added to compensate the macro-misalignments. Bearing in mind that relative movement between the back and the shoulder centre is the result of a somewhat complex mechanism, it is very difficult to design a mechanism that is kinematically equivalent to a human and capable of fitting different users. Therefore, an end-point-based solution is proposed, comprising a four-bar mechanism with longitudinal sliders for displacements along the shoulder plane and a perpendicular prismatic joint with two cylindrical sliders (see Figure 4).

This configuration allows all the torque to be transmitted to the back via the exoskeleton so that the shoulder does not have to exert the corresponding forces. This solution reduces shoulder muscle fatigue, making the extended or flexed arm position a case of equivalent load from the viewpoint of the forces transmitted to the human body.

The range of movement achieved with this mechanism is wide enough to compensate for the three-directional movement of the instantaneous centre of rotation of the

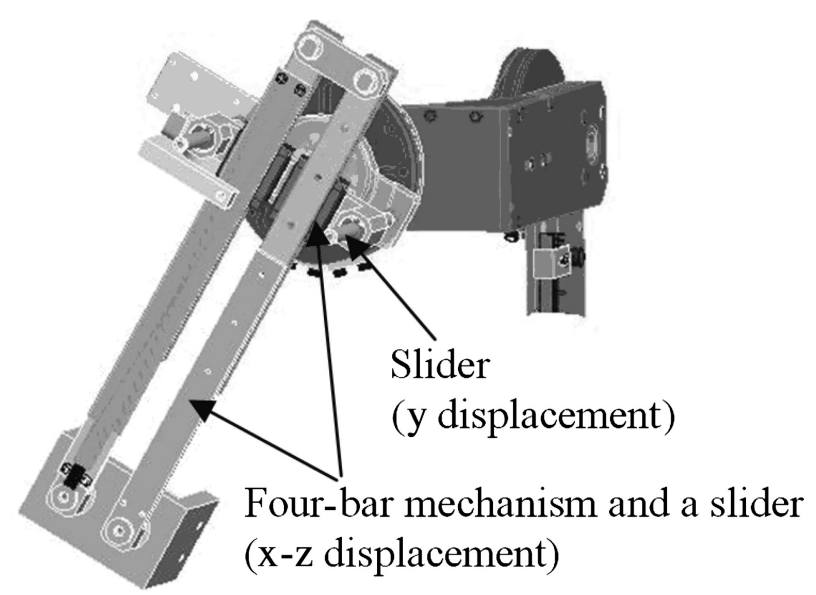

Figure 4. Auxiliary DOF at shoulder. glenhumeral shoulder joint during shoulder elevation along a sagittal plane.

The design of the four-bar mechanism and the longitudinal sliders must take into account the fact that both sliders cannot be aligned with the perpendicular direction of the bars within the range of movement; if they are aligned, a singular point appears.

Apart from the above-mentioned DoF, the design also has an additional passive DoF for compensating micromisalignments between the elbow and the wrist. This DoF is necessary to prevent injury, as the centre of rotation of the exoskeleton may not coincide with that of the arm and its position changes throughout the workspace. In this case, the traction/compression forces are transmitted by the arm and the other forces and movements are transmitted by the exoskeleton. In specific cases, this degree of freedom may also be blocked.

\subsection{IKO's kinematic ranges}

The various ranges of movement of each DoF of the exoskeleton taken into account in the design specifications are summarised in Table 1. It also shows the ranges limited by software to guarantee the safety of the exoskeleton, which are more restrictive than those that can be physically reached.

\section{Mechanical design and first prototype}

\subsection{Actuation solution proposal}

Two alternatives were considered with respect to the solution for the actuation system: placing the actuators directly on each joint, or placing them on an external base and transmitting the forces through an additional transmission system.

The first option has a major disadvantage: as the actuators move with the exoskeleton, mass and inertia are considerably increased, together with the power requirements of the DoF of the shoulder. Figure 5 shows the torque requirements at elbow flexion joint for two hypotheses: case 1 , considering only arm and exoskeleton structure; and case 2 , also considering the mass of the elbow rotation actuator placed at the wrist. In case 2, torque requirements at elbow flexion increase up to twice as much as in case 1.

Table 1. Motion ranges for the exoskeleton.

\begin{tabular}{lcclcr}
\hline & \multicolumn{2}{c}{ Design range $\left(^{\circ}\right)$} & & \multicolumn{2}{c}{ Allowed range $\left(^{\circ}\right)$} \\
\cline { 2 - 3 } \cline { 5 - 6 } \cline { 5 - 6 } & Min & Max & & Min & Max \\
\hline Shoulder abduction & -70 & 160 & & -30 & 115 \\
Shoulder flexion & -61 & 187 & & -30 & 175 \\
Shoulder rotation & -71 & 108 & & -60 & 80 \\
Elbow flexion & 0 & 149 & & 5 & 110 \\
Forearm rotation & -97 & 104 & & -80 & 80 \\
\hline
\end{tabular}




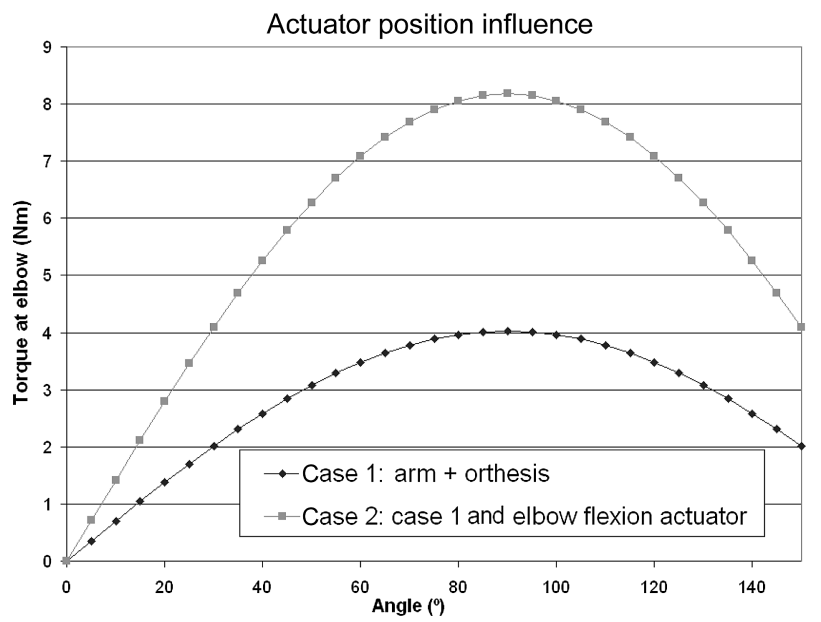

Figure 5. Torque requirements at elbow flexion DoF.

Furthermore, the space occupied by the actuators can limit the range of movement, the exoskeleton's workspace and the dynamic performance.

Taking these limitations into account, the decision was taken to place all the actuators in a backpack joined to the exoskeleton by the auxiliary DoF as mentioned in Section 2.2 .

Movement transmission is made by a pair of sheathed cables (Bowden cables) from the backpack to the joints. For this solution, the total weight of the backpack and all the actuators is $13.3 \mathrm{~kg}$, and the moving mass is reduced considerably, with a weight of $3.7 \mathrm{~kg}$ for the five actuated DoF of the arm and the passive DoF for avoiding micromisalignments between the wrist and the elbow joint. In addition, the weight of auxiliary passive DoF at shoulder is $1.6 \mathrm{~kg}$, but the movements of this mechanism are very small, especially when shoulder elevation does not exceed $90^{\circ}$. These masses could be reduced by decreasing the $10-$ $\mathrm{kg}$ lifting requirement, especially if the exoskeleton is to be used for rehabilitation.

The cables provide great flexibility of movement and also enable a mechanical fuse function, limiting the maximum force each joint can exert to protect the user from overstrain. In the case of this prototype, cables with a configuration of $1 \times 19$ and diameters of $1.2 \mathrm{~mm}(1700 \mathrm{~N}$ ultimate strength) were used for third and fifth DoF, and cables with a diameter of $1.6 \mathrm{~mm}(2270 \mathrm{~N})$ were used for the others.

The only drawback of transmitting the movement from the motor to the joint via a Bowden cable is the efficiency reduction due to friction forces between cable and sheath. The losses caused by friction can be estimated by taking into account the bend angle and friction coefficient between the cable and the sheath (Goiriena et al. 2009; Letier et al. 2008).

$$
T=W e^{\mu \theta}
$$

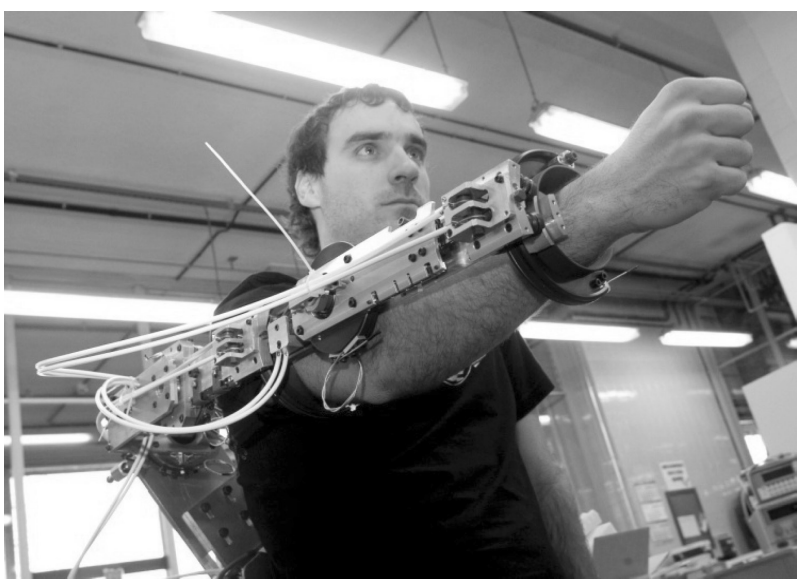

Figure 6. The five-DoF exoskeleton prototype IKO (front).

where $T$ is the tension needed to lift a light weight at a constant velocity, $\mu$ is the coefficient of kinetic friction between the cable and the sheath and $\theta$ is the bend angle of the sheath. The friction coefficient must be estimated for each application taking into account the pair contact between the sheath and the cable. For our application, these values have been measured obtaining a value of 0.11 for 1.2 $\mathrm{mm}$ cable diameter and 0.125 for a $1.6-\mathrm{mm}$ cable diameter. These values were used to define cable requirements and dimensioning.

Figures 6 and 7 show pictures of the resulting five actuated DoF exoskeleton prototype IKO. All the actuators and their corresponding drivers and regulators are arranged on two plates placed on top of each other and resulting in a compact-size backpack (Figure 7).

The proposed design can be used as an internal force device when the backpack is worn by the user or as an external force device when the backpack is attached to an external element, e.g. a chair.

\subsection{Actuator configuration and selection}

According to the literature, most exoskeleton developments have been designed and implemented using direct current (DC) motors due to the fact that they are easily integrated, high powered, low cost and easily controlled. In any case, major advances are being made today in the development of artificial muscles in order to replicate the biological qualities of animal muscles. As one of the requirements, Ikerlan wanted to use non-conventional actuators and special work was carried out with pneumatic muscles to research their applicability in industrial applications and, more specifically, in the development of orthoses and wearable robots.

The pneumatic muscle - the biologically inspired artificial muscle - was invented in the 1950s by the physician Joseph L. McKibben to actuate an arm orthosis so as to help 


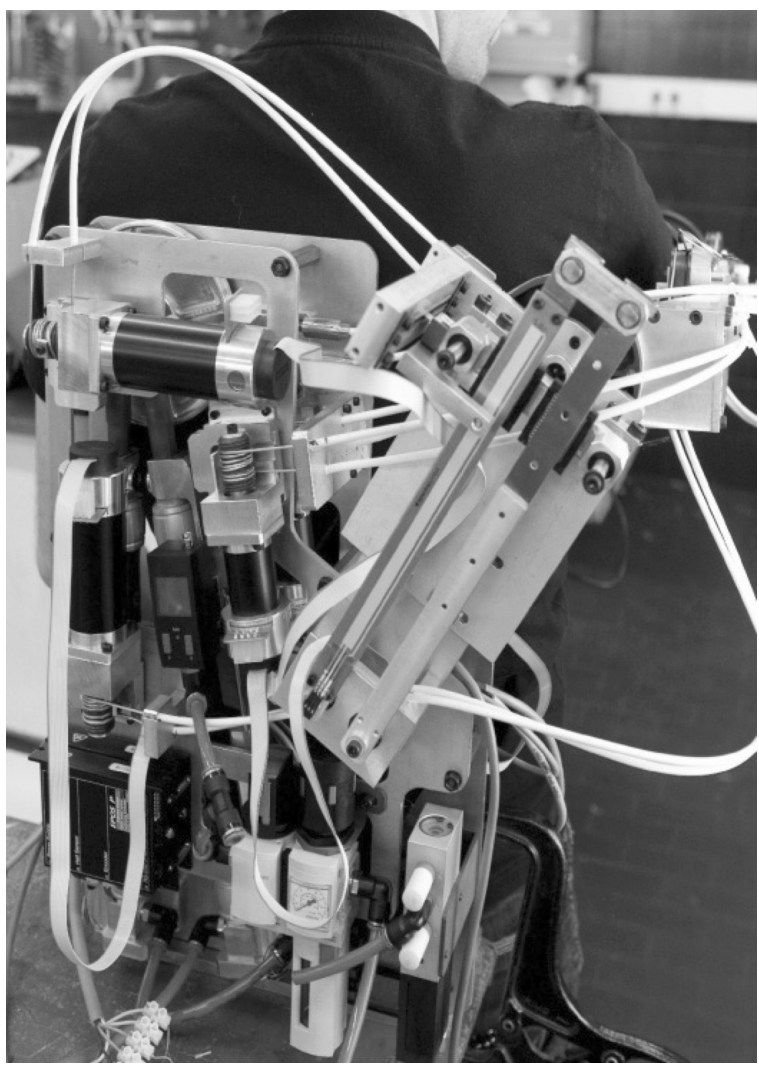

Figure 7. The five-DoF exoskeleton prototype IKO (back).

control a disabled hand (Tondu and Lopez 2000). Although this type of actuator subsequently fell into oblivion, interest in it was revived at the end of the twentieth century due to its high-performance actuating features, good power/weight ratio and lightness, which enables the easy design of robotic applications with low impedance in terms of their interaction with the environment. The manufacturers Shadow and Festo market industrial versions of the McKibben muscle.

Unfortunately, pneumatic artificial muscles evidence certain very non-linear force-length characteristics, like animal muscles, and controlling them and obtaining the performance features demanded by some applications are not easy issues. The modelling of pneumatic muscles and controller design have been active research issues in recent years (Colbrunn et al. 2001; Petrovic 2002; Schröder et al. 2003; Tondu and Lopez 2000).

As a first step, an experimental one-DoF arm powered by pneumatic muscles manufactured by Festo was designed and constructed at Ikerlan. The initial work was developed to obtain a model of the complete system in the Dymola/Modelica package, the modelling and simulation tool used as standard in Ikerlan, for which a model of a pneumatic muscle has been made.

The objective of the pneumatic muscle model is to find a relation between the pressure and length of the pneumatic muscle with the force it exerts along its entire axis. As has been mentioned before, the model has been implemented by using the positive qualities of the modelling language of physical systems known as Modelica (Pujana-Arrese et al. 2007).

Thus, certain experiments and in-simulations were performed, obtained both in open- and closed-loop mode, as a way to validate the models and the modelling procedure. Based on the non-linear model, linear models were then obtained (Pujana-Arrese et al. 2007).

However, the specification of the orthosis being able to grasp and hold a maximum weight of $10 \mathrm{~kg}$ limited the applicability of pneumatic muscles in most of the DoF. As a result, conventional electrical motors were used for four DoF and pneumatic muscles for only one DoF for the forearm rotation.

The electrical motor used, which was the same for the four DoF, was the Maxon EC45 flat 50W KL, with an encoder MR of 500 pulses per turn. The gearheads used were Maxon GP32C with a reduction of 111 for the elbow flexion and the shoulder rotation; and Maxon GP42C with a reduction of 319 for the shoulder flexion and abduction. As a result and taking into account the gearheads and pulleys, the total reductions are 540 for the elbow flexion, 781 for the shoulder rotation and 1562 for the shoulder rotation and abduction.

In order to minimise the space required, Maxon EPOS P 24/5-positioning controllers are used to drive the electrical motors. Although the position set-point generation and the position-control algorithms are performed externally, the velocity set-point values that are generated are sent to the controllers through the analogical ports and converted to the required units.

The elbow rotation is powered by two DMSP-20$200 \mathrm{~N}$ pneumatic muscles manufactured by Festo, where the length of the muscle fibre is $200 \mathrm{~mm}$ when they are not under pressure. The muscles are controlled by a Festo MPYE-5-1/8HF pneumatic servovalve with a working pressure of six bar. Festo SDE-D10 pressure sensors are used to establish the entry pressure of each muscle. The length of each muscle is measured by a Crouzet $3 \mathrm{k} \Omega$ linear sensor. After the cable pulley transmission, the relationship between the muscles and the elbow angle is $10.67^{\circ} / \mathrm{mm}$.

Although they are not needed for control, redundant linear potentiometers have been implemented at the load tip of each DoF for safety reasons and to help estimate the absolute position of the elements.

\section{Aesthetic redesign}

Most wearable exoskeletons have been designed taking into account criteria of kinematic compatibility between devices and the human body, the miniaturisation of actuators and sensors for lightening weight and movement accuracy. Nevertheless, as these devices have to be worn by humans, aesthetic criteria must also be considered. Perception of the 
friendless and the safety perceived by the user and by the environment are as important as a product's functionality. Nowadays, most developments have a robot design with metal or dark colours, in search of an image of a technologically advanced system. Aesthetics must take into account not only the user wearing the exoskeleton but also the people in the area in which it is going to move, i.e. assistance personnel and other workers in the case of industrial applications.

Even if these criteria are subjective, depending on the origin, there are certain issues that can improve aesthetics:

- Design, rounded shapes and edges, function and element integration of designs, making simpler, more compact designs. Design must be as similar as possible to the human body and must observe proportionality between the parts.

- Surface texture can be used to transmit safety or other sensations to the user.

- Colour combination can be used for the same design, making it suitable for different applications such as industry, rehabilitation, domestic use or even products for men or women (Heller 2008).

The design and colour issues applied in the redesigning of IKO's first prototype have been taken into account from two points of view: mechanism redesign and cover design. Textures have not been considered because their implementation is quite expensive for smallsize series. The workplace, i.e. a chair, has also been designed.

\subsection{Mechanism redesign}

Based on the preliminary design, an improved redesign process was carried out. The main purpose was to reduce the number of parts and take aesthetics into account.

Most of the parts needed to support sensors or Bowden cable transmission have been implemented in existing parts, greatly reducing the number of small parts and, consequently, fastening screws. Structural parts have been reshaped to keep the proportion between different parts. In the search of an intuitive design, parts involved in circular movements at the shoulder and elbow are circular in shape, integrating the design of the sheaves at the points where the Bowden cable acts. Figure 8 shows a picture of the final proposal. Overall, the total mass of the system has been reduced by $0.4 \mathrm{~kg}(15 \%)$.

\subsection{Cover design}

The previous approach is a good solution for designing mass custom products that have to be cheap, but its implementation in preliminary prototypes is very expensive. Another solution is to design a cover that hides most of the functional parts and can be used to address different feelings. Some of the advantages include its easy modification, even with regard to shape and colours. For aesthetic purposes, parts can be created directly using a 3-D plotter. These covers can be easily fitted to existing designs.

The proposed cover design consists of four parts: two for the shoulder-to-elbow section and another two for the elbow-wrist section. These two pairs of parts allow a relative longitudinal displacement and relative rotation. Covers are circular in shape to fit with the external shape of the arm and extensions have been made to hide the circular sliders.
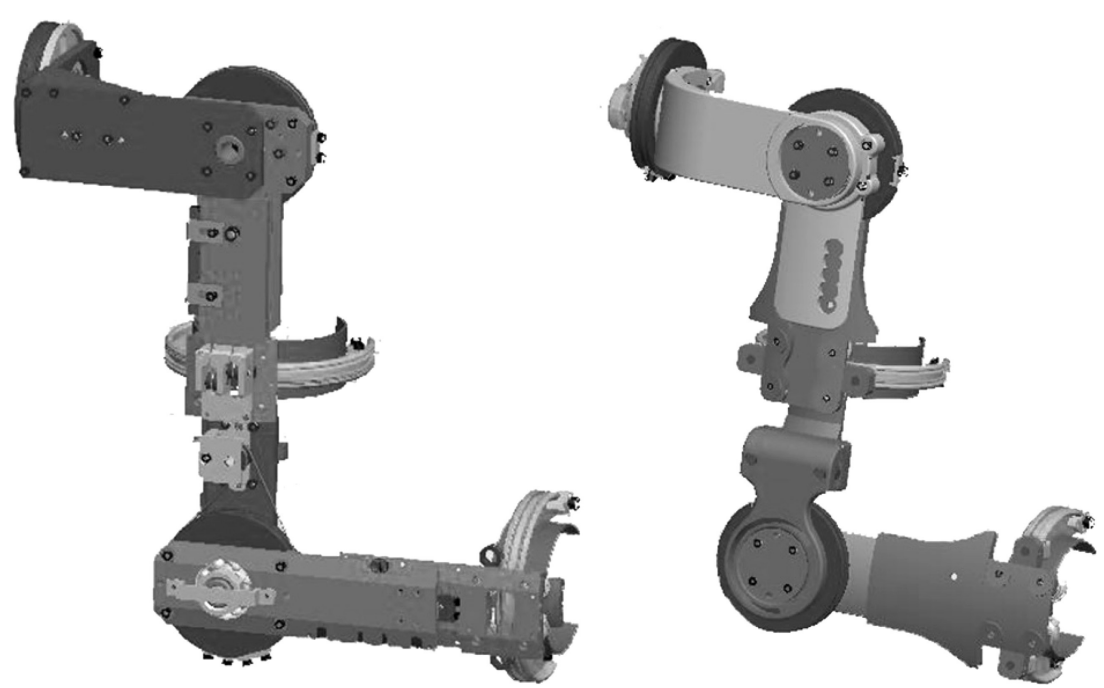

Figure 8. Proposal of redesigned mechanism. 

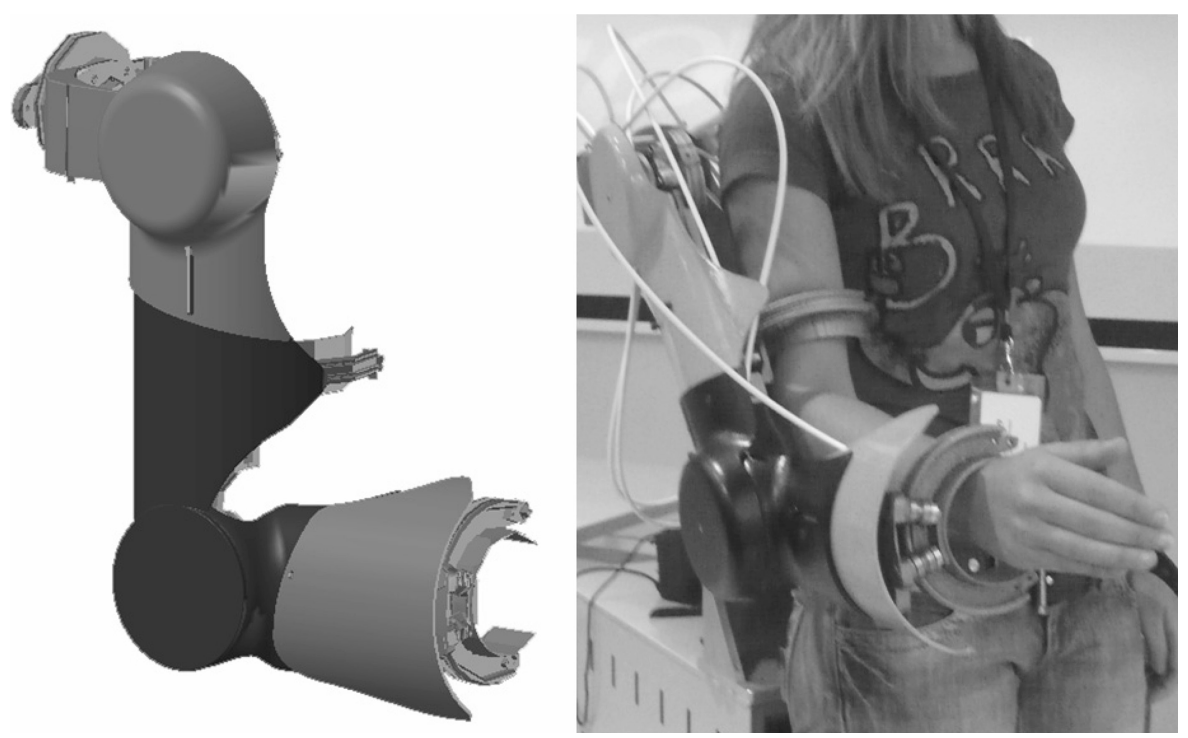

Figure 9. Cover designed and constructed.

As the cover is for aesthetics only, it does not support any force and can be fastened to the exoskeleton via clips so that it can be changed with ease. This makes it possible to adapt the exoskeleton to different applications.
The initial ideas for designing the cover included simple structures that follow the shapes of the human arm, transmit harmony and stay away from the designs of robots or machines. In other words, it must be a
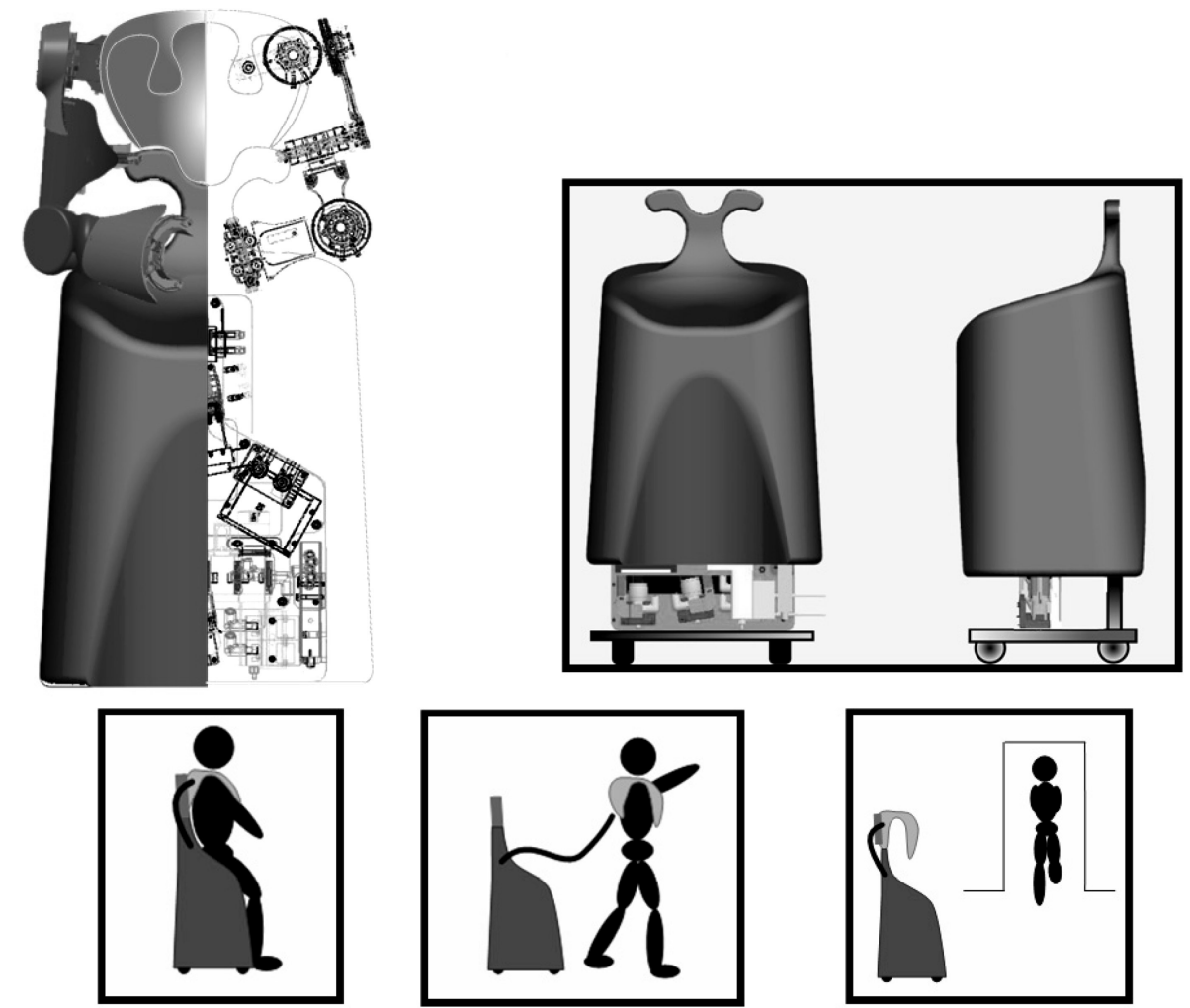

Figure 10. Workplace designed. 


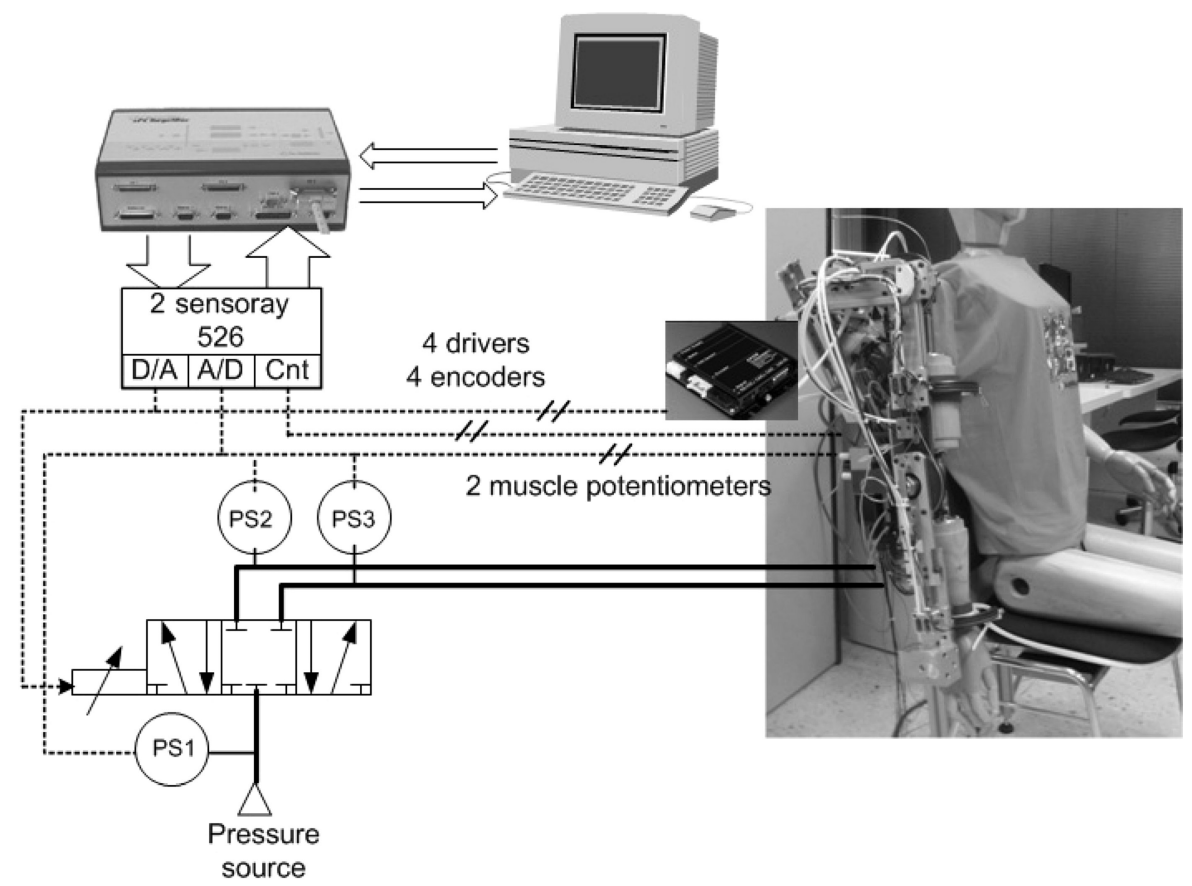

Figure 11. Schematic diagram of IKO controller.

user-friendly product and transmit confidence and safety when it is worn. Different colours have been used for each part of the cover, depending on their mechanical operation. Figure 9 shows the cover as it was finally designed and built. The mass added to the system by this cover is $0.4 \mathrm{~kg}$.

\subsection{Workplace design}

Besides the communication function, consideration has also been given to the use from the utiliser's standpoint, i.e. it has to be easy to use at the workplace. Accordingly, a hypothetical future design has been made: consideration has been given to a workplace that is a chair, where the user can stand or sit down. For both cases, the functions of the backpack have been transferred to the specially designed chair. If the chair is on wheels, it can be moved easily from one place to another. When the work is completed, the exoskeleton can be fastened to the chair and take up little space. Figure

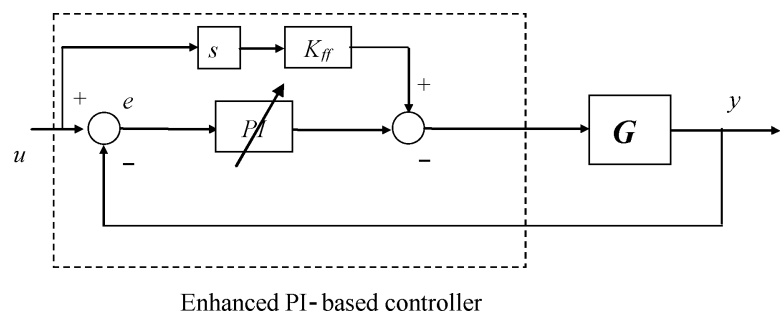

Figure 12. Forearm rotation controller structure.
10 shows the hypothetical working environment around the specially designed chair.

\section{Control structure and experimental results}

\subsection{Control structure}

IKerlan's Orthosis hardware system from the control point of view is shown in Figure 11. As described in Section 3.2, to operate the five actuated DoF, DC motors are used, except for the elbow rotation, which uses pneumatic muscles. The motors are controlled by Maxon EPOS P 24/5, which have been programmed to accept speed set-point values. The pneumatic muscles are controlled by the Festo servovalve. The position controllers are closed on the control hardware. As shown in the figure, the control hardware is PIP8, an industrial personal computer (PC) made by the company

Table 2. Values of PI parameters used in the forearm rotation controller.

\begin{tabular}{lll}
\hline & $K_{p}$ & $K_{i}$ \\
\hline Zone $\left[0^{\circ} / 20^{\circ}\right]$ & 0.026 & 0.031 \\
Zone $\left[20^{\circ} / 40^{\circ}\right]$ & 0.021 & 0.024 \\
Zone $\left[40^{\circ} / 60^{\circ}\right]$ & 0.029 & 0.0243 \\
Zone $\left[60^{\circ} / 80^{\circ}\right]$ & 0.022 & 0.022 \\
Zone $\left[0^{\circ} /-20^{\circ}\right]$ & 0.026 & 0.031 \\
Zone $\left[-20^{\circ} /-40^{\circ}\right]$ & 0.021 & 0.024 \\
Zone $\left[-40^{\circ} /-60^{\circ}\right]$ & 0.029 & 0.0243 \\
Zone $\left[-60^{\circ} /-80^{\circ}\right]$ & 0.022 & 0.022 \\
\hline
\end{tabular}




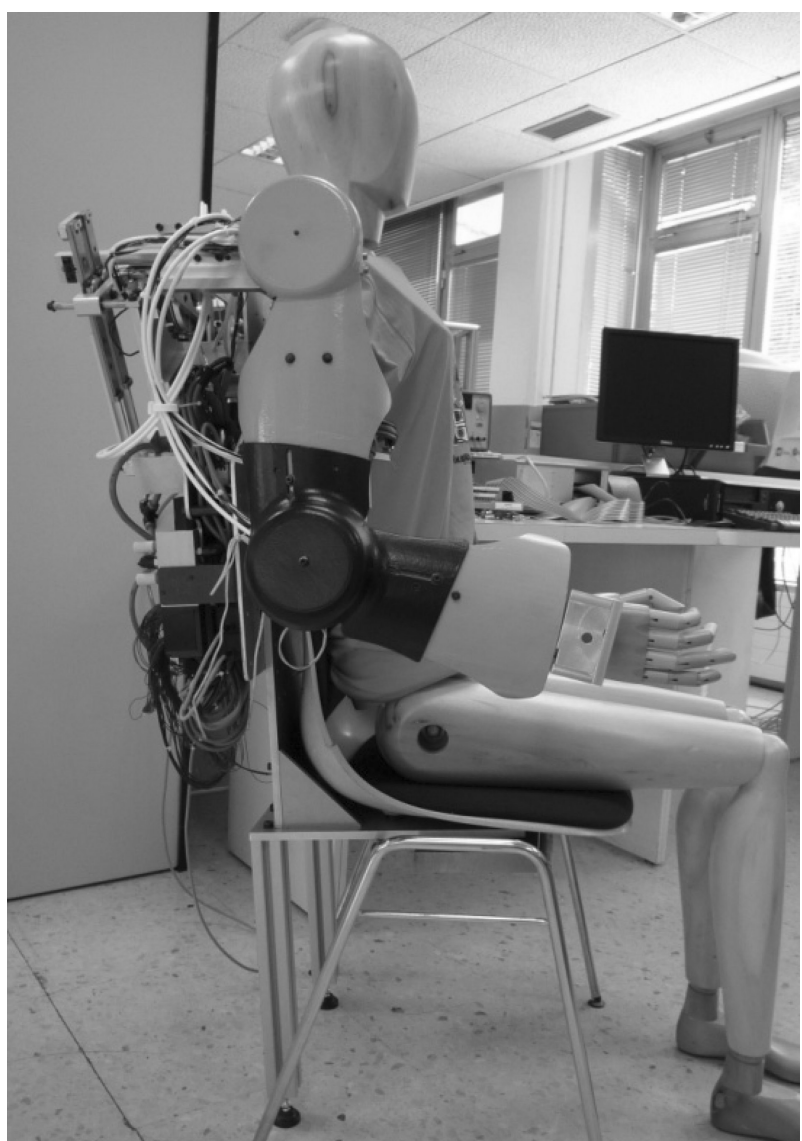

Figure 13. Exoskeleton IKO worn by a dummy.
MPL, which is very similar to The MathWorks' $x$ PCTargetBox. By means of two PC104 cards (sensory model 256) inserted into the PIP8, all the control signals can be read and written. Control algorithms are implemented in Simulink and the code is generated and downloaded to the aforementioned hardware by means of two of The MathWorks tools: $R T W$ and $X P C$ Target.

Despite the fact that the figure shows only the signals involved in the position control of the five actuated DoF, as described in Section 3.2, there are many more sensors whose signals are acquired and used in the control algorithms. On the one hand, there are the sensors of the three passive DoF of the shoulder and on the other there is also a position sensor on the load of each DoF for safety reasons and to help estimate the absolute position of the elements. Initially, force and torque sensors have not been included, but they will be included in the future.

From the point of view of the position control strategies, taking into account the resulting total reductions, the axes powered by electrical motors are very stiff and a Pposition controller is robust enough for load changes. However, the control of the pneumatic muscles is different. Their impedance is low but they are very non-linear and difficult to control properly. Research was carried out on the position control of pneumatic muscles, where different algorithms, from an enhanced PID-based controller to $\mathrm{H}_{\infty}$ and sliding-mode robust control techniques, were applied and compared (Pujana-Arrese et al. 2008).

Figure 12 shows the position controller finally designed and implemented for the pneumatically actuated forearm rotation of IKO. Basically, it consists of a PI algorithm, tuned up in different angle ranges and implemented with a gain-scheduling strategy. A speed

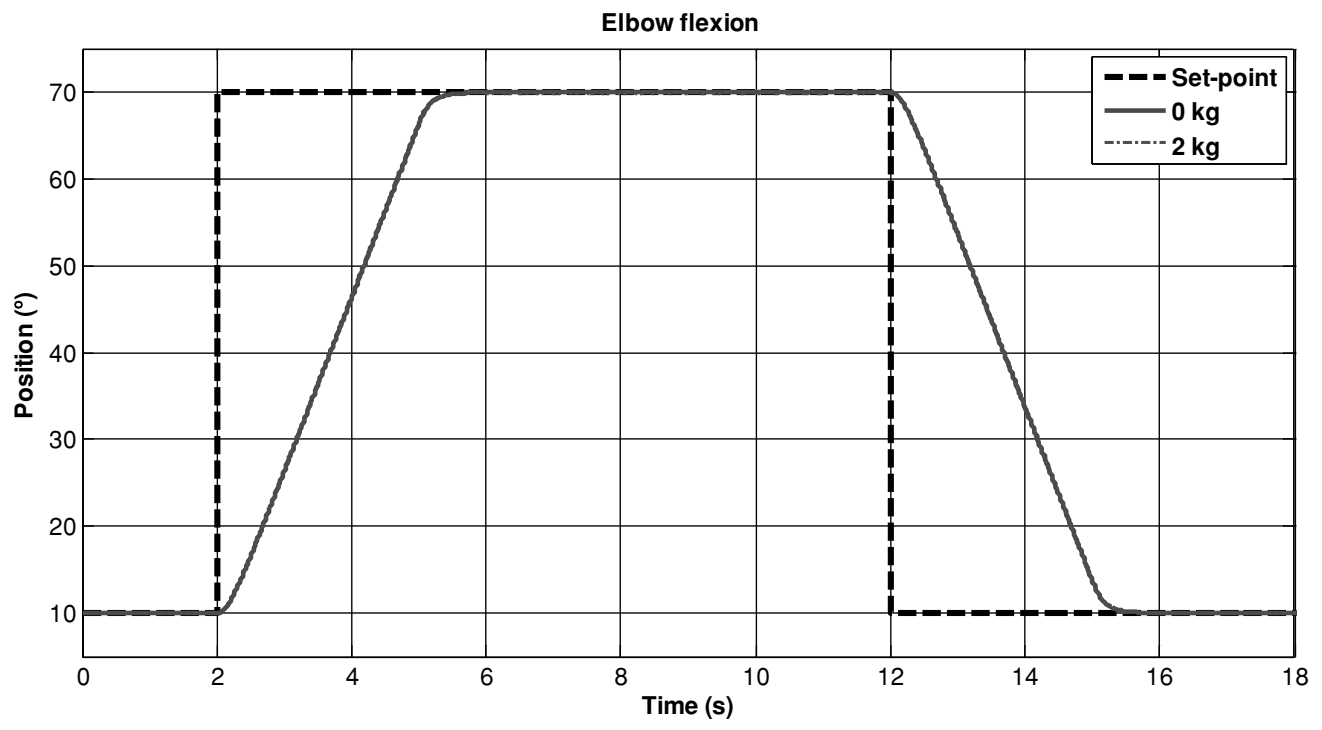

Figure 14. Experimental results in elbow flexion. 


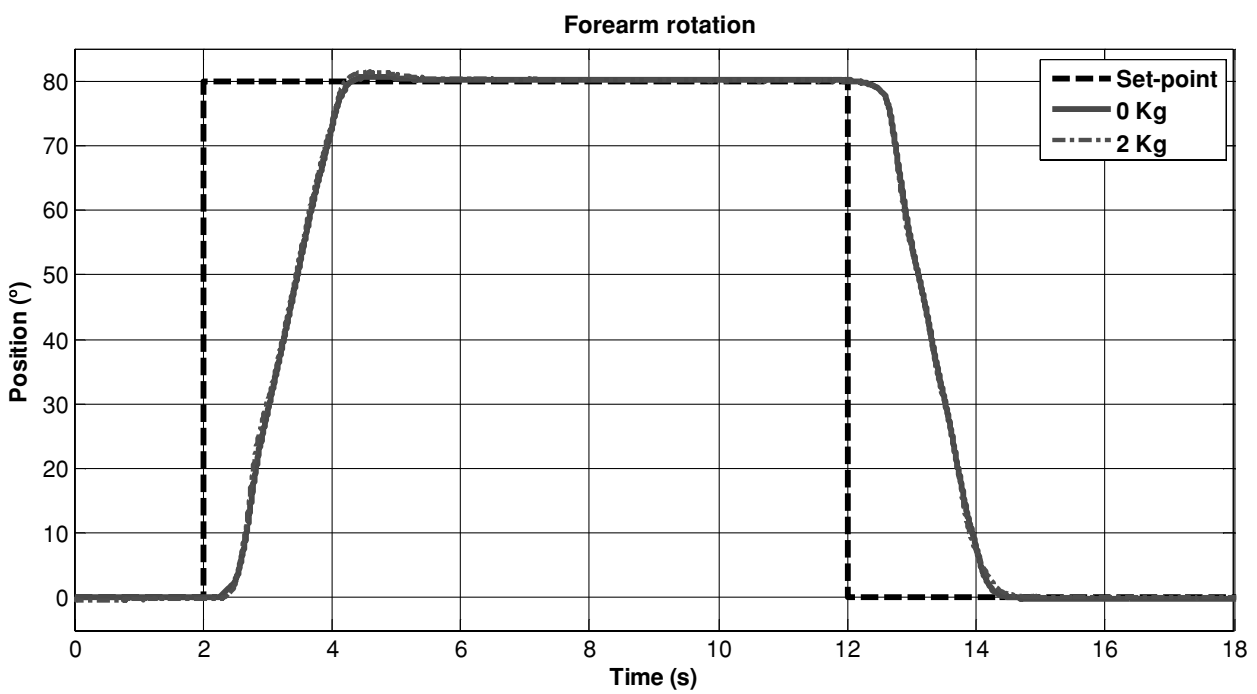

Figure 15. Experimental results in forearm rotation.

feedforward was also added to improve the dynamic response.

Owing to the non-linearity of the antagonistic pair of pneumatic muscles, it was decided to divide the $160^{\circ}$ wristrotation movement range into eight operating zones and tune up the controller parameters for each zone. In view of the fact that, with regard to the rest position, the system shows the same behaviour in both turn directions, it is suffice to carry out the tuning-up process only for the four zones of one side of the range. Table 2 shows the values of $K_{i}$ and $K_{p}$ that optimise the controller response in each operating area. For the feedforward loop a gain of $K_{f f}=$ 0.0021 was used.

\subsection{Experimental results}

Experimental results are given to show the dynamic features of the actuated DoF. Initially, in order to perform the movements, a dummy wore the exoskeleton as shown in Figure 13. To see the influence of a mass grasped by

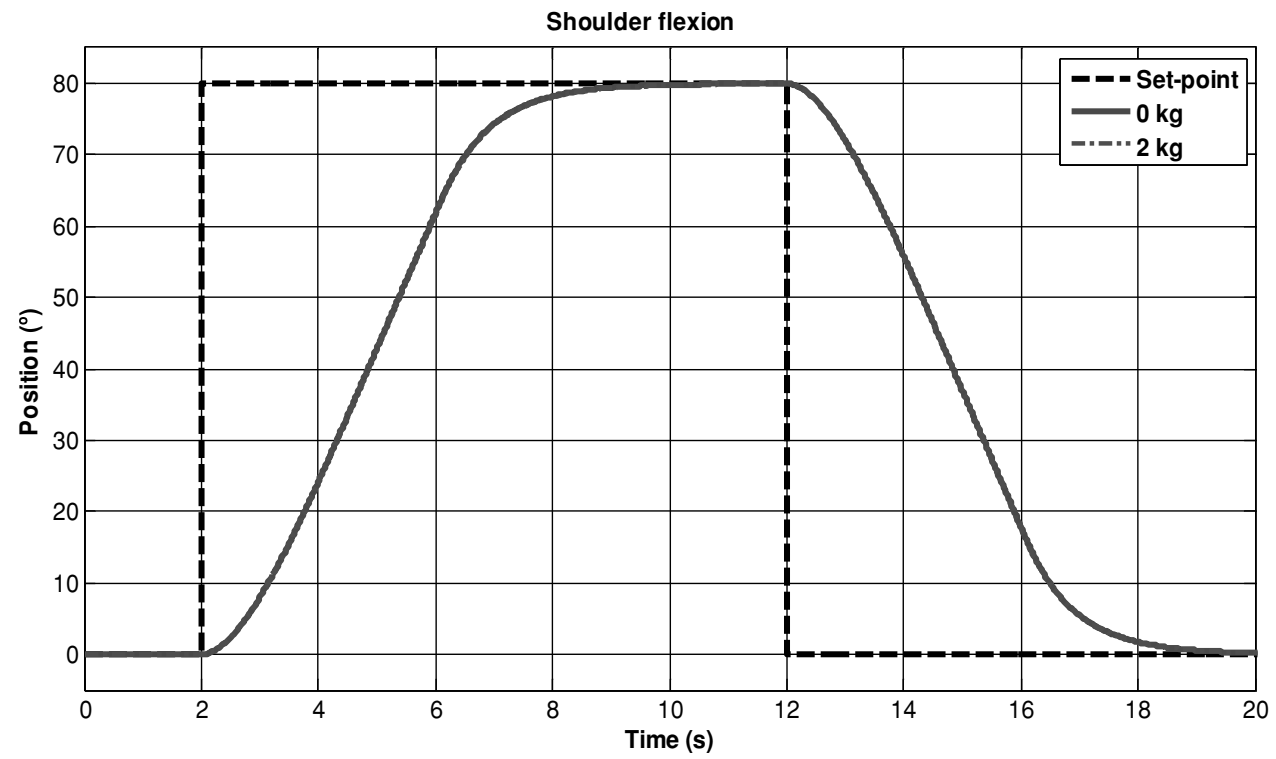

Figure 16. Experimental results in shoulder flexion. 
the hand, a weight of $2 \mathrm{~kg}$ was fixed to the dummy's hand. Figure 14 shows the response of the elbow flexion to a $60^{\circ}$ upward jump followed by a downward jump, first of all with no weight in the hand and then with a $2-\mathrm{kg}$ weight. Firstly, despite the action of gravity, which is not expressly compensated, the upward and downward dynamics are seen to be identical. Furthermore, there is absolutely no difference when the weight is placed in the hand. The time required for movement is around $3.5 \mathrm{~s}$ due to the motor that is used and the resulting reduction factor.

Figure 15 shows the results of an experiment performed with forearm rotation, with no mass grasped by the hand and then with 2-kg weight. It was rotated $80^{\circ}$ to the left and then to the right. It was observed that the stationary is reached in around $2 \mathrm{~s}$ and that the steadystate error is very small, although the movement dynamics are quicker than in the case of flexion. Positioning with the pneumatic muscles is within a margin of $\pm 0.5^{\circ}$ in all the zones. The dynamics obtained on rotation to the left and to the right is also very similar, although there are slight differences due to different friction. The influence of the hand mass in the dynamic response is very small.

Figure 16 shows the response of the shoulder flexion with the arm completely extended (elbow flexion angle fixed at the minimum value) to an $80^{\circ}$ upward jump followed by a downward jump, with and without mass in the hand. Owing to the adaptation, the dynamics are a bit slower than in the case of elbow flexion, but the mass grasped by the hand has no influence.

\section{Conclusions and next stages}

This paper presents IKO, a wearable five-DoF upper limb exoskeleton for increasing performance levels during daily tasks, especially at the workplace. The selection of the five actuated DoF and certain auxiliary passive DoF was based on both ergonomic considerations and maximum reachable workspace. Conventional electrical motors and pneumatic muscles are used for actuation purposes. Bowden cable-drive transmission appears to be a good solution for reducing moving mass and actuator and structural part size. After the first prototype was built, a redesign process was carried out, where materials and, in particular, aesthetic issues were analysed in detail. For the next stages, an inverse kinematic model is being set up and tested in simulation before it is integrated in IKO, together with a generator of position set-point values. There are certain important topics that are being analysed in more detail: ergonomic issues, mechanism impedance, Bowden cable durability and efficiency and interaction with the user and the environment. Another very important issue we are working on is the detection of the user's intention.

\section{Acknowledgement}

The material used in this paper was partly supported by the Spanish Ministry of Education and Science and European Regional Development Fund (research project DPI2006-14928-C02-01).

\section{References}

Akella P, Peshkin M, Colgate JE. 1999. Cobots for the automobile assembly line. Paper presented at: IEEE International Conference on Robotics and Automation, Detroit.

Colbrunn RW, Nelson GM, Quinn RD. 2001. Modeling of braided pneumatic actuators for robotic control. Paper presented at: International Conference on Intelligent Robots and Systems (IROS'01), Maui, HI.

Goiriena A, Retolaza I, Cenitagoya A, Martinez F, Riaño S, Landaluze J. 2009. Analysis of bowden cable transmission performance for orthosis applications. Paper presented at: 5th IEEE International Conference on Mechatronics, ICM 2009, Malaga (Spain).

Heller E. 2008. Psicología del color [Psychology of colour]. Gustavo Gil: SL, Barcelona, Spain. ISBN: 978-84-252-1977-1.

Klug S, von Stryk O, Mohl B. 2006. Design and control mechanisms for a 3DoF Bionic manipulator. Paper presented at: IEEE International Conference on Biomedical Robotics and Biomechatronics-BioRob'06, Pisa, Italy.

Letier P, Avraam M, Veillerette S, Horodinca M, De Bartolomei M, Schiele A, Preumont A. 2008. SAM: a 7-DoF portable arm exoskeleton with local joint control. Paper presented at: IEEE/RSJ International Conference on Intelligent Robots and Systems, Sep, 22-26, Nice, France.

Martinez F, Retolaza I, Lecue E, Basurko B, Landaluze J. 2007. Preliminary design of an upper limb IAD (intelligent assist device). AAATE Conference, Oct 3-5, San Sebastian, Spain.

NASA. 2008. Available from: http://msis.jsc.nasa.gov/sections/ section03.htm.

Perry JC, Rosen J. 2006. Design of a 7 degree-of-freedom upper-limb powered exoskeleton. Paper presented at: IEEE International Conference on Biomedical Robotics and Biomechatronics-BioRob'06, Pisa, Italy.

Petrovic PB. 2002. Modeling and control of an artificial muscle. 10th Conference on Mechanical Vibrations (CVM 2002) 23-24 May 2002, Timosoara, Romania.

Podniece Z. 2007. Work-related musculoskeletal disorders: back to work report. European Agency for Safety and Health at Work. Available from: http://osha.europa. eu/publications/reports/7807300.

Pons JL. 2008. Wearable robots: biomechatronic exoskeletons. San Francisco, CA: Wiley \& Sons. ISBN 978-0-470-51294-4, cap. 8, 2008

Pujana-Arrese A, Arenas J, Maruri L, Martinez-Esnaola A, Landaluze J. 2007. Modelling and validation of a 1-DOF arm powered by pneumatic muscles. Paper presented at: Industrial Simulation Conference ISC'2007, June 11-13, Delft, Holland.

Pujana-Arrese A, Riaño S, Arenas J, Martinez-Esnaola A, Landaluze J. 2008. $\mathrm{H}_{\infty}$ position control of a 1-DoF arm powered by pneumatic muscles. Paper presented at: 8 th Portuguese Conference on Automatic Control-CONTROLO'2008, July 21-23, Vila Real (Portugal).

Ruiz AF, Forner-Cordero A, Rocon E, Pons JL. 2006. Exoskeletons for rehabilitation and motor control. Paper presented at: IEEE International Conference on Biomedical Robotics and Biomechatronics-BioRob'06, Pisa, Italy. 
Schiele A, van der Helm FCT. 2006. Kinematic design to improve ergonomics in human machine interaction. IEEE Trans Neural Syst Rehabil Eng. 14(4):456469.

Schröder J, Erol D, Kawamura K, Dillmann R. 2003. Dynamic pneumatic actuator model for a model-based torque controller. Proceedings of the IEEE International Symposium on Computational Intelligence in Robotics and Automation. 16-20 July 2003.

Tondu B, Lopez P. 2000. Modeling and control of McKibben artificial muscle robot actuators. IEEE Contr Syst Mag. April:15-38. 

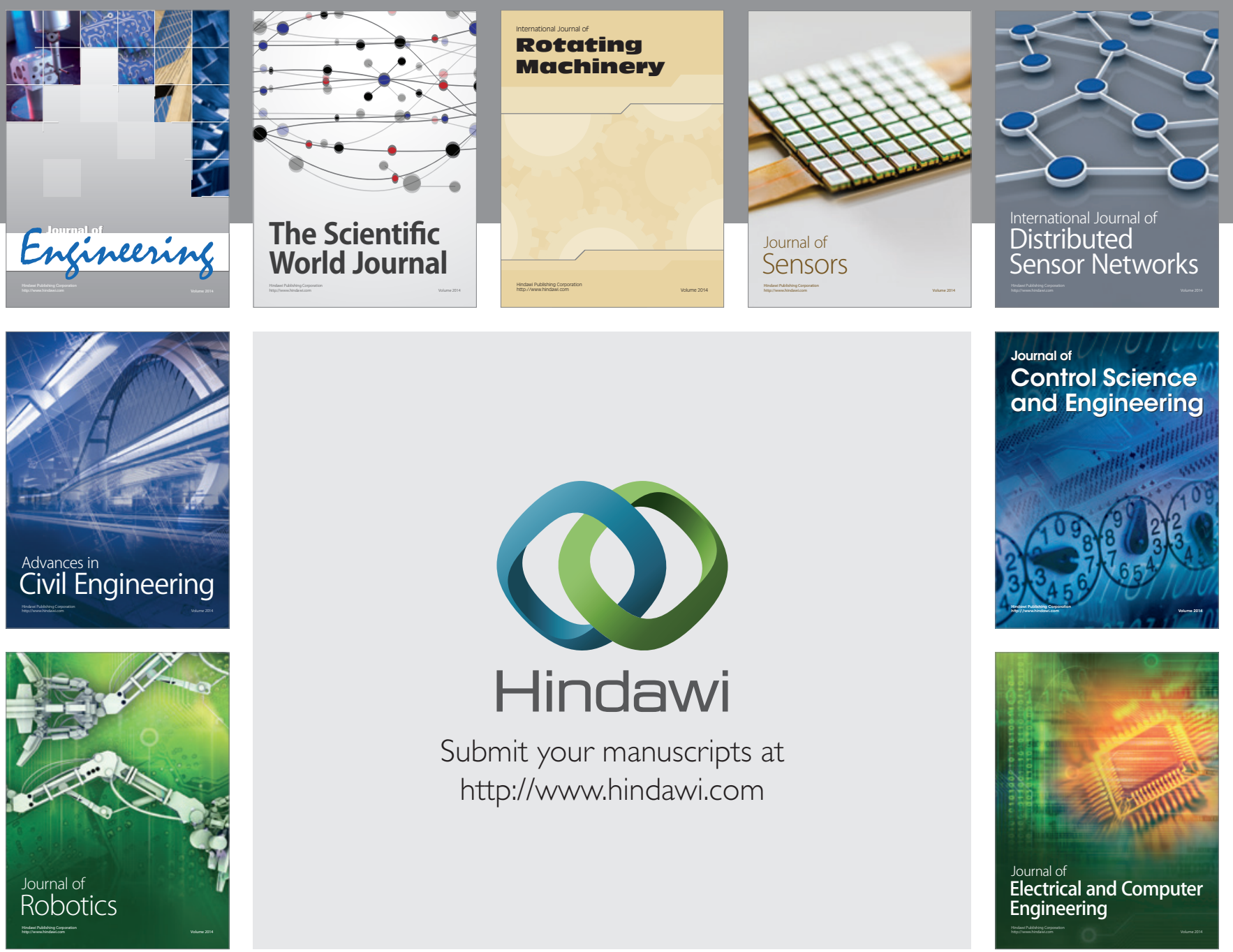

Submit your manuscripts at

http://www.hindawi.com
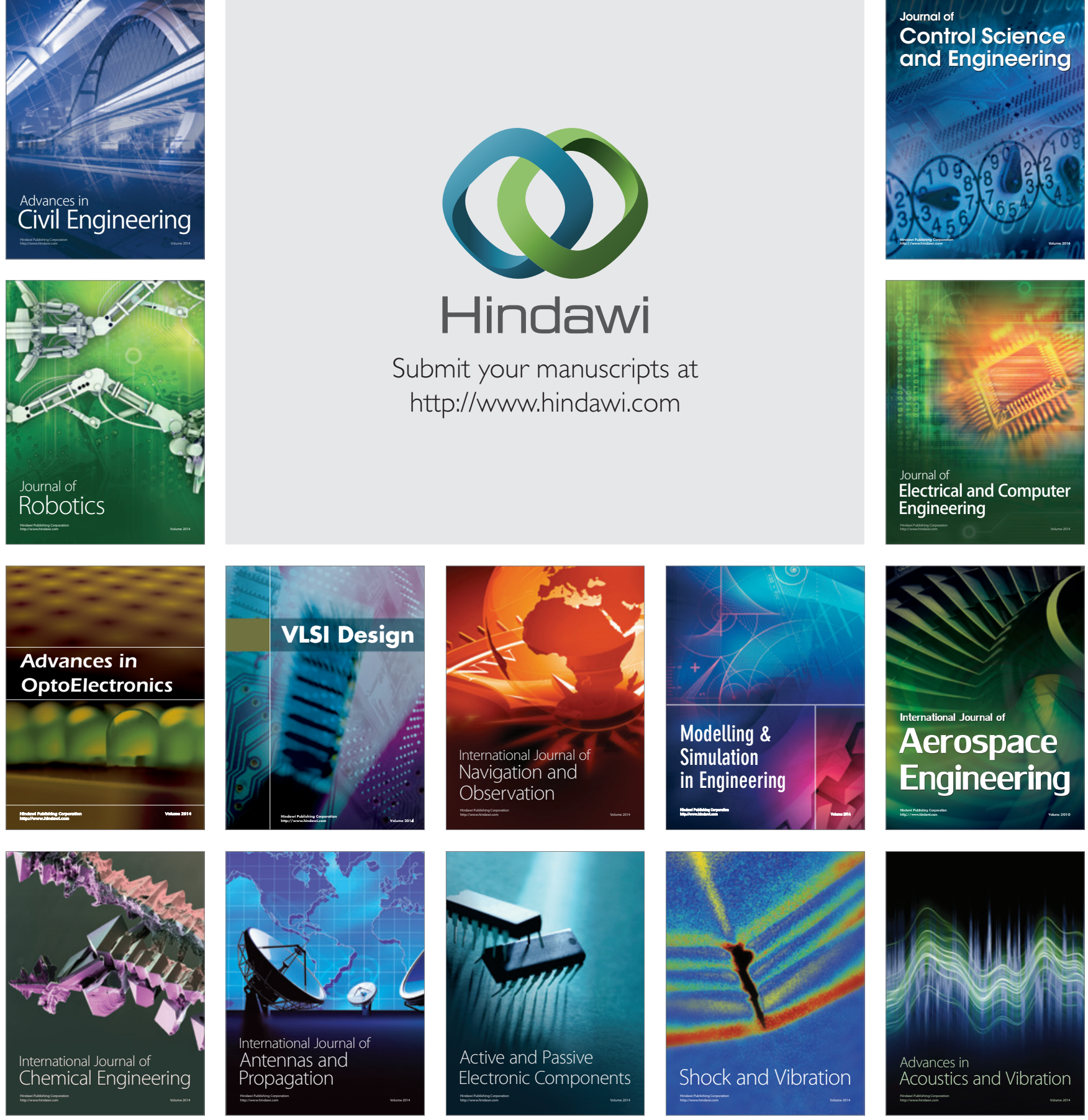